Cahiers $d u$ MONDE RUSSE

\section{Cahiers du monde russe}

Russie - Empire russe - Union soviétique et États indépendants

$59 / 4 \mid 2018$

Varia

\title{
Polina Barskova, Besieged Leningrad
}

Aesthetic Responses to Urban Disaster

Sarah Gruszka

\section{(2) OpenEdition}

1 Journals

Édition électronique

URL : https://journals.openedition.org/monderusse/10722

DOI : $10.4000 /$ monderusse. 10722

ISSN : $1777-5388$

Éditeur

Éditions de l'EHESS

\section{Édition imprimée}

Date de publication : 1 octobre 2018

Pagination : $645-648$

ISBN : 978-2-7132-2747-9

ISSN : 1252-6576

\section{Référence électronique}

Sarah Gruszka, « Polina Barskova, Besieged Leningrad », Cahiers du monde russe [En ligne], 59/4 | 2018, mis en ligne le 01 octobre 2018, consulté le 08 janvier 2022. URL : http://journals.openedition.org/ monderusse/10722 ; DOI : https://doi.org/10.4000/monderusse.10722 


\section{Polina BARSKOVA}

\section{Besieged Leningrad}

\section{Aesthetic Responses to Urban Disaster}

De Kalb : NYU Press, 2017, 232 p.

Cette dernière décennie a vu se renouveler considérablement les études sur le siège de Leningrad (1941-1944), cet épisode tragique de la guerre germano-soviétique durablement mythifié par l'historiographie soviétique. Pas une année ne se passe sans qu'un ouvrage fondamental ne paraisse sur le sujet, en Russie ou dans le monde anglo-saxon et germanique, qu'il soit l'œuvre d'historiens ou de spécialistes de littérature ${ }^{1}$. Professeur de littérature russe aux États-Unis, Polina Barskova fait partie des chercheurs les plus actifs sur la question ${ }^{2}$. Dans la bouillonnante historiographie du siège, elle se distingue par une approche essentiellement littéraire, basée sur l'analyse des textes. Dans son dernier ouvrage, elle s'intéresse à l'expression et à l'esthétisation de la douleur du siège, abordées selon un angle pour le moins original, celui des « réactions des assiégés à leur situation spatiale » et de leur « imagination urbaine » (p. 193). Ce faisant, elle cherche à déterminer dans quelle mesure les conditions d'existence infra-humaines modifient le rapport des individus à leur environnement physique et culturel et, plus largement, à comprendre les modalités d'adaptation de celui qu'elle nomme « le sujet assiégé », ce Léningradois affaibli, transi, affamé, à l'une des plus grandes catastrophes du $\mathrm{XX}^{\mathrm{e}}$ siècle. Polina Barskova s'inscrit ainsi dans la lignée des recherches qui se sont penchées sur les raisons et les stratégies de survie dans Leningrad assiégé, une question qui ne cesse d'être débattue par les historiens, le corps médical et la société civile 3 .

L'auteur envisage Leningrad assiégé comme un espace de crise sociale, militaire et politique, où le trauma historique produit non seulement de l'indicible, un certain mutisme, mais aussi une forme de créativité à travers la quête de nouvelles ressources langagières à même de surmonter le défi de l'irreprésentabilité et de convertir l'expérience tragique en écriture. Pour étayer son propos, elle s'appuie sur des sources diversifiées : prose et poésie (publiées ou non) et journaux intimes contemporains à l'événement principalement, complétés de quelques entretiens menés avec des survivants, des œuvres picturales et cinématographiques, ainsi que certains écrits officiels, idéologiques, en regard desquels elle étudie parfois les textes produits par les assiégés. On peut d'emblée regretter une classification parfois sommaire des sources, comme celle qui revient à nommer « journal intime » des souvenirs (par définition rétrospectifs), ou encore l'imprécision de certaines références archivistiques, de même que celle des citations de journaux intimes (la mention de la date d'écriture aurait été souhaitable). Comme en témoigne la richesse de la bibliographie, Polina Barskova puise également dans la culture mondiale littéraire et philosophique, en convoquant Gaston Bachelard, Jurij Lotman, Roland Barthes, Ernst Bloch, Henri Lefebvre, Walter Benjamin (notamment pour sa notion de « désordre créateur »), etc., et en recourant à des concepts tels que l'ekphrasis (la description d'images visuelles par des moyens textuels), la défamiliarisation (notion empruntée à Viktor Šklovskij), ou encore l'hétérotopie (Michel Foucault). 
C'est forte de ce bagage culturel et toujours sous l'angle de la «spatialité traumatique » (p. 193) de la ville assiégée que l'auteure aborde, en six chapitres, différents aspects de l'identité ou de l'existence altérées par le blocus : le déplacement, le corps, la vue, la lecture. Dans le premier chapitre, consacré à la mobilité urbaine, elle interroge le nouveau rapport à l'environnement que construisent les assiégés désorientés par la défiguration de Leningrad, en se focalisant sur trois mouvements de la marche (la flânerie, le cercle, le retour) et son dialogue avec l'espace et la mémoire urbaine. Elle s'appuie notamment sur deux concepts situationnistes, la « dérive » et la « psychogéographie » (les effets psychiques du contexte urbain sur l'individu, p. 24). Le deuxième chapitre interroge les représentations allégoriques des corps squelettiques des Léningradois affamés, dont Polina Barskova révèle l'ambivalence, la « cacophonie » (p. 51), parfois la contradiction (les corps sont tantôt repoussants, tantôt héroïques). Le troisième chapitre porte sur « les paradoxes de la vision du siège », notamment sur le défi de la représentation discursive ou visuelle (par l'art) des ténèbres (Leningrad assiégé étant plongé dans l'obscurité en raison des pénuries de carburant). L'auteur en vient à envisager l'aveuglement comme producteur de sensibilités inédites et d'un nouveau savoir (p. 91).

Dans le chapitre suivant, l'un des plus étendus, Polina Barskova explore l'irruption paradoxale de la beauté au milieu de l'horreur du siège. Selon elle, le sujet assiégé, paralysé voire insensible à l'horreur (sous les effets d'un épuisement extrême) tend à l'esthétiser, élaborant par là une véritable stratégie de survie mentale à même de combler le vide de la perte et d'atténuer les souffrances (p. 98, 132). Partant d'un concept kantien, elle forge la notion de « sublime du siège » qu'elle définit comme l'ensemble des procédés de dissimulation esthétique de l'expérience du trauma - que l'on peut retrouver dans le contexte concentrationnaire. Son analyse la conduit à considérer la représentation urbaine du siège comme un phénomène esthétique particulièrement fécond. Leningrad assiégé serait ainsi non seulement un lieu d'enfermement, de chaos et de destruction, générant fréquemment un sentiment de captivité et d'angoisse, mais aussi un matériau stimulant la créativité et l'émergence de nouvelles perceptions.

Les deux derniers chapitres sont consacrés à la lecture pendant le siège de Leningrad. Après avoir rappelé les usages multiples de la matérialité du livre dans les conditions de pénurie, Polina Barskova explore les mécanismes qui sous-tendent la lecture pendant le siège. En répondant à la question « quels livres étaient lus pendant le siège, comment et pourquoi ? », l'auteur identifie différentes fonctions thérapeutiques et constructives de la lecture : elle permet l'évasion, à la fois spatiale et temporelle, hors de la situation de crise dans laquelle les assiégés sont enfermés (ce qu'elle appelle les « directions hétéchroniques » de la lecture, p. 150), et elle délivre des clés à même d'éclairer leur vécu déroutant. Polina Barskova révèle tout un réseau intertextuel où des modèles littéraires (Puškin, Tolstoj, Proust, Poe, Dickens...) sont mobilisés, réactivés par les Léningradois, pour nourrir leurs propres représentations du siège. Elle suggère ainsi que le recours à des œuvres littéraires familières peut aider les sujets d'une catastrophe urbaine à créer un espace mental, une bulle protectrice qui faciliterait la survie (p. 153). 
Dans l'épilogue, l'auteur s'intéresse à ce qu'elle appelle le travail de " post-mémoire », c'est-à-dire le regard de nos contemporains qui ont cherché à restituer l'espace assiégé, à travers l'étude de deux productions filmiques (dont Blokada [Le siège] de Sergej Loznica, sorti en 2005) et d'une installation artistique récente, dont elle souligne l'apport au regard du canon narratif soviétique. Détectant un besoin traumatique de réexpérimenter des perturbations sensorielles historiques, elle avance la notion d' " écho du siège » et d' " écho-construction » (p. 179).

Polina Barskova conclut à l'émergence d'une poétique du siège, qui génère ses propres références, « un vaste réseau complexe de représentations culturelles » (p. 193), son propre langage avec ses tropes spécifiques permettant la mise en mots d'une expérience qui semblait précisément échapper à toute représentation. Tout en déconstruisant un certain nombre de stéréotypes du canon narratif soviétique (comme les effets bénéfiques de la radio), elle revisite l'un de ses topoï, à savoir la permanence de la culture et de l'art pendant le blocus, sans pour autant occulter l'effet déshumanisant de la famine. Tissant des parallèles avec d'autres catastrophes historiques et se nourrissant de travaux portant sur le trauma, l'auteur confère à l'expérience du siège une portée transcendant largement le cadre de l'événement.

Accompagné d'un index et d'un appareil critique précieux, Besieged Leningrad. Aesthetic Responses to Urban Disaster apporte un éclairage inédit sur la culture du siège de Leningrad, à condition, néanmoins, de posséder déjà de solides connaissances sur cet épisode que l'auteur, qui ne prétend pas faire œuvre d'historien, ne s'attarde pas toujours à contextualiser. Il reste à espérer que cet ouvrage contribuera à stimuler l'intérêt pour le siège de Leningrad qui, selon Polina Barskova, demeure encore, plus de soixante-dix ans après, un événement historique «non grata » (p. 188).

1 - Parmi les plus stimulants, signalons Richard Bidlack, Nikita Lomagin, The Leningrad Blockade, 1941-1944 : a New Documentary History from the Soviet Archives, New Haven : Yale University Press, 2012 ; Сергей Яров, Повседневная жизнь блокадного Ленинграда [Sergej Jarov, La vie au quotidien pendant le siège de Leningrad], M. : Molodaja Gvardija, 2013 ; Manfred Sapper, Volker Weichsel, éds., Die Leningrader Blockade Der Krieg, die Stadt und der Tod, Berlin : Osteuropa, 2011 ; Alexis Peri, The War Within : Diaries from the Siege of Leningrad, Cambridge - London : Harvard University Press, 2017 ; et, tout récemment, Татьяна Воронина, Помнить по-намему. Соиреалистический историзм и блокада Ленинграда [Tat'jana Voronina, Se souvenir : l'historisme social-réaliste et le siège de Leningrad], M. : Новое литературное обозрение, 2018.

2 - Outre les nombreux articles dont elle est l'auteur, signalons l'ouvrage collectif qu'elle a récemment co-dirigé : Полина Барскова, Риккардо Николози, ред., Блокадные нарративы. Сборник статей [Polina Barskova, Riccardo Nikolosi, éds., Histoires du siège recueil d'articles], M. : Novoe literaturnoe obozrenie, 2017, ainsi que l'édition de deux anthologies de textes écrits pendant le siège de Leningrad : Written in the Dark: Five Poets in the Siege of Leningrad, New York: Ugly Duckling Press, 2016 et Блокада. Свидетельства о Ленинградской блокаде. Хрестоматия [Le siège : Témoignages sur le siège de Leningrad. Morceaux choisis], M. : Izdatel'skij proekt « A i B », 2017. Poétesse reconnue, elle a enfin publié plusieurs recueils de poèmes en russe et en anglais. 3 - Sur cet aspect du siège, voir Джон Барбер, Андрей Дзенискевич, ред., Жизнь и смерть в блокированном Ленинграде: Историко-медииинский аспект [Vivre 
et mourir pendant le siège de Leningrad : aspects historico-médicaux], SPb. : Dmitrij Bulanin, 2001 ; Jeffrey Hass, « Norms and Survival in the Heat of War: Normative Versus Instrumental Rationalities and Survival Tactics in the Blockade of Leningrad », Sociological forum, 26 (4), 2011, p. 921-949 ; Геннадий Соболев, Ленинград в борьбе за выживание в блокаде (в 3-х книгах) [Gennadij Sobolev, Leningrad dans la lutte pour la survie pendant son siège (en 3 vol.)], SPb. : Izd-vo Sankt-Peterburgskogo gos. Universiteta, 2013-2017.

Sarah Gruszka

Sorbonne Université Lettres 